\title{
NONSEPARABILITY AND UNIFORM STRUCTURES IN LOCALLY COMPACT GROUPS
}

\author{
G. HANSEL AND J. P. TROALLIC
}

(Communicated by Roe Goodman)

\begin{abstract}
Let $G$ be a locally compact topological group. We prove that if $G$ is not a SIN-group, then the quotient Banach space $\mathscr{U}_{L}(G) / \mathscr{U}(G)$ contains an isometric linear copy of $l \infty$. To get this result, we first establish an extension theorem for (bilaterally) uniformly continuous functions on $G$.
\end{abstract}

\section{INTRODUCTION}

Let $G$ be a topological group and $C(G)$ the usual Banach space of all continuous bounded complex-valued functions on $G$. Let $\mathscr{A}$ and $\mathscr{B}$ be two distinct Banach subspaces of $C(G)$ with $\mathscr{B} \subset \mathscr{A}$. In such a framework, a now standard question is to ask if the quotient Banach space $\mathscr{A} / \mathscr{B}$ is nonseparable or even contains an isometric linear copy of the space $l^{\infty}$ of bounded complex sequences. C. Chou [2], [3] and H. A. M. Dzinotyiweyi [4], [5] have established several interesting results of this type and the main purpose of this paper is to present a new one.

As in [13], let us denote by $\mathscr{U}_{L}^{\prime}(G)$ (respectively $\mathscr{U}_{R}(G)$ ) the space of all the functions in $C(G)$ which are left (respectively right) uniformly continuous and let $\mathscr{U}(G)=\mathscr{U}_{L}(G) \cap \mathscr{U}_{R}(G)$ be the space of all functions in $C(G)$ which are (bilaterally) uniformly continuous. In [9] G. L. Itzkowitz has shown that if $G$ is a nonunimodular locally compact topological group, then $\mathscr{U}_{L}(G)$ and $\mathscr{U}(G)$ are distinct, and H. A. M. Dzinotyiweyi [4] has asked if the quotient $\mathscr{U}_{L}(G) / \mathscr{U}(G)$ is nonseparable. Liater on P. Milnes [13] has extended the result of G. L. Itzkowitz by showing that $\mathscr{U}_{L}(G)$ and $\mathscr{U}(G)$ are distinct as soon as $G$ is not a SIN-group, i.e., if its two usual uniform structures are different (recall that a locally compact SIN-group is unimodular). Hence the question of $\mathrm{H}$. A. M. Dzinotyiweyi is relevant in this case. In the third section of the present work, we show that in fact if $G$ is not a SIN-group, then $\mathscr{U}_{L}(G) / \mathscr{U}(G)$ contains an isometric linear copy of $l^{\infty}$.

To get this result we first establish an extension theorem for (bilaterally) uniformly continuous functions on $G$. The method of proof is similar to that

Received by the editors September 4, 1992 and, in revised form, August 13, 1993.

1991 Mathematics Subject Classification. Primary 22D05; Secondary 54E15, 46E15.

Key words and phrases. Locally compact groups, uniform structures, uniformly continuous functions. 
of the classical Urysohn's lemma. Let us remark that it does not seem possible to deduce this theorem from Katetov's extension theorem of bounded uniformly continuous functions [11], [12].

\section{BilATERALLY UNIFORMLY CONTINUOUS EXTENSIONS}

Let $G$ be a topological group and let $e$ be its identity element. As in [8], a complex-valued function $f$ on $G$ is called left uniformly continuous if it is uniformly continuous with respect to the left uniform structure on $G$, i.e., if for any $\varepsilon>0$ there is a neighbourhood $V$ of $e$ in $G$ such that for $x, y \in G$,

$$
x^{-1} y \in V \Longrightarrow|f(x)-f(y)|<\varepsilon \text {. }
$$

As said above, we denote the Banach space of bounded left uniformly continous functions on $G$ by $\mathscr{U}_{L}(G)$; the space $\mathscr{U}_{R}(G)$ of bounded right uniformly continuous functions on $G$ is defined accordingly with respect to the right uniform structure of $G$, and we denote the Banach space of bounded (bilaterally) uniformly continuous functions on $G$ by $\mathscr{U}(G)$.

A finite sequence $\left(W_{1}, \ldots, W_{m}\right)$ of neighbourhoods of $e$ is called well fitted if there is a neighbourhood $X$ of $e$ in $G$ such that $X W_{i} X \subset W_{i+1}$ for all $i=1, \ldots, m-1$.

The following two lemmas are quite simple: they only use elementary properties of neighbourhoods of the identity in a topological group.

Lemma 2.1. Let $\left(W_{1}, W_{2}, \ldots, W_{m}\right)$ be a well fitted finite sequence of neighbourhoods of $e$ in $G$. Then there is a finite sequence $\left(W_{1}^{\prime}, \ldots, W_{m-1}^{\prime}\right)$ of neighbourhoods of $e$ in $G$ such that the "mixed" finite sequence $\left(W_{1}, W_{1}^{\prime}, \ldots, W_{m-1}\right.$, $\left.W_{m-1}^{\prime}, W_{m}\right)$ is well fitted.

Proof. Let $X$ be a neighbourhood of $e$ in $G$ such that $X W_{i} X \subset W_{i+1}$ for all $i=1, \ldots, m-1$. Let $Y$ be a neighbourhood of $e$ in $G$ such that $Y^{2} \subset$ $X$. For all $i=1, \ldots, m-1$, let $W_{i}^{\prime}=Y W_{i} Y$; then the finite sequence $\left(W_{1}^{\prime}, \ldots, W_{m-1}^{\prime}\right)$ satisfies the required condition.

Lemma 2.2. Let $W$ be a neighbourhood of $e$ in $G$ and let

$$
D=\left\{\frac{i}{2^{n}} \mid n \in \mathcal{N}, i=0,1 \ldots, 2^{n}\right\}
$$

be the set of all rational positive dyadic numbers in $[0,1]$. Then there is a $D$ indexed family $\left(W_{t}\right)_{t \in D}$ of neighbourhoods of $e$ in $G$ such that $W_{1}=W$ and such that for all $n \in \mathscr{N}$ the finite sequence

$$
\left(W_{\frac{0}{2^{n}}}, W_{\frac{1}{2^{n}}}, \ldots, W_{\frac{2^{n}}{2^{n}}}\right)
$$

of neighbourhoods of $e$ in $G$ is well fitted.

Proof. We define the sets $W_{\frac{i}{2^{n}}}, i=0,1, \ldots, 2^{n}$, by induction on $n$. Let $W_{1}=W$ and let $W_{0}$ be a neighbourhood of $e$ in $G$ such that $\left(W_{0}, W_{1}\right)$ is well fitted. Suppose that a well fitted sequence

$$
\left(W_{\frac{0}{2^{n}}}, W_{\frac{1}{2^{n}}}, \ldots, W_{2^{n}}\right)
$$

of neighbourhoods of $e$ in $G$ has already been built. Then according to Lemma 2.1 , for any rational dyadic number $\frac{2 j+1}{2^{n+1}}, j=0, \ldots, 2^{n}-1$, we 
can choose a neighbourhood $W_{\frac{2 j+1}{2^{n+1}}}$ of $e$ in $G$ such that the sequence

$$
\left(W_{\frac{0}{2^{n+1}}}, W_{\frac{1}{2^{n+1}}}, \ldots, W_{\frac{2^{n+1}}{2^{n+1}}}\right)
$$

is well fitted. tion.

The following result is the "extension theorem" announced in the introduc-

Theorem 2.3. Let $\left(A_{n}\right)_{n \in \mathscr{N}}$ be a sequence of subsets of $G$, and let $c=\left(c_{n}\right)_{n \in \mathscr{N}} \in$ $l^{\infty}$. Suppose there is a neighbourhood $Z$ of $\dot{e}$ in $G$ such that the elements of the sequence $\left(Z A_{n} Z\right)_{n \in \mathscr{N}}$ are pairwise disjoint. Then there is a function $h \in \mathscr{U}(G)$ such that $h(x)=c_{n}$ for all $x \in A_{n}, n \in \mathscr{N}$.

Proof. If $c=0$, the null function of $\mathscr{U}(G)$ satisfies the required conditions. So let us suppose that $c \neq 0$. Let $W$ be a neighbourhood of $e$ in $G$ such that $W^{2} \subset Z$ and let $\left(W_{t}\right)_{t \in D}$ be a $D$-indexed family of neighbourhoods of $e$ in $G$ whose existence is asserted by Lemma 2.2. Let us put

$$
A=\bigcup_{n \in \mathscr{N}} W A_{n} W
$$

Remark that the sets $W A_{n} W, n \in \mathscr{N}$, are pairwise disjoint; indeed the sets $Z A_{n} Z, n \in \mathcal{N}$, are pairwise disjoint and for all $n \in \mathscr{N}$, we have $W A_{n} W=$ $\left(Z A_{n} Z\right) \cap A$.

For all $x \in A$, let us define $t_{x} \in[0,1]$ and $c_{x} \in \mathscr{C}$ in the following way: let $n$ be the positive integer such that $x \in W A_{n} W$; we put

$$
t_{x}=\inf \left\{t \in D \mid x \in W_{t} A_{n} W_{t}\right\} \text { and } c_{x}=c_{n} .
$$

Then we define the function $h: G \rightarrow \mathscr{C}$ by

$$
h(x)= \begin{cases}c_{x}\left(1-t_{x}\right) & \text { for all } x \in A, \\ 0 & \text { for } x \in G \backslash A .\end{cases}
$$

With this definition, it is clear that $h(x)=c_{n}$ for all $x \in A_{n}, n \in \mathcal{N}$, and moreover $h$ is bounded. Let us show that $h$ is left uniformly continuous.

Let $\varepsilon>0$. Let $p \in \mathscr{N}$ be such that $\frac{1}{2^{p-1}} \leq \frac{\varepsilon}{\|c\|}$ and let $X_{p} \subset W$ be a symmetrical neighbourhood of $e$ in $G$ such that for $i=0,1, \ldots, 2^{p}-1$

$$
X_{p} W_{\frac{i}{2^{p}}} X_{p} \subset W_{\frac{i+1}{2^{p}}}
$$

Let $x, y \in G$ be such that $x^{-1} y \in X_{p}$ and let us show that

$$
|h(x)-h(y)| \leq \varepsilon
$$

If the points $x$ and $y$ belong to $G \backslash A$, then $h(x)=h(y)=0$. So let us suppose that at least one of the points $x$ and $y$ belongs to $A$.

First case: $x$ and $y$ belong to $A$.

Let $m$ be the positive integer such that $x \in W A_{m} W$; then we get that $y \in W A_{m} W$ as a consequence of the inclusions

$$
y \in x X_{p} \subset W A_{m} W X_{p} \subset W A_{m} W^{2} \subset Z A_{m} Z .
$$

It follows that $c_{x}=c_{y}=c_{m}$ and consequently

$$
|h(x)-h(y)|=\left|c_{m}\right|\left|t_{x}-t_{y}\right| \leq\|c\||| t_{x}-t_{y} \mid \text {. }
$$


Hence to get inequality $(1)$, we have only to show that $\left|t_{x}-t_{y}\right| \leq \frac{1}{2^{p-1}}$. Suppose that $t_{x}<t_{y}$. Let $i \in\left\{0,1, \ldots, 2^{p}-1\right\}$ be such that

$$
\frac{i}{2^{p}} \leq t_{x}<\frac{i+1}{2^{p}}
$$

If $i+2>2^{p}$, we have

$$
\left|t_{x}-t_{y}\right|=t_{y}-t_{x} \leq 1-\frac{i}{2^{p}}=\frac{2^{p}-i}{2^{p}}<\frac{1}{2^{p-1}} .
$$

If $i+2 \leq 2^{p}$, since

$$
y \in x X_{p} \subset W_{\frac{i+1}{2 p}} A_{m} W_{\frac{i+1}{2 p}} X_{p} \subset W_{\frac{i+2}{2 p}} A_{m} W_{\frac{i+2}{2 p}},
$$

we have $t_{y} \leq \frac{i+2}{2^{p}}$ and consequently

$$
\left|t_{x}-t_{y}\right|=t_{y}-t_{x} \leq \frac{i+2}{2^{p}}-\frac{i}{2^{p}}=\frac{1}{2^{p-1}} .
$$

Second case: $\{x, y\} \not \subset A$. Let us suppose that $x \in A$ and $y \in G \backslash A$. Let $m$ be the positive integer such that $x \in W A_{m} W$. We have

$$
|h(x)-h(y)|=|h(x)|=\left|c_{m}\right|\left(1-t_{x}\right) \leq\|c\|\left(1-t_{x}\right) .
$$

Hence to get inequality (1), we have only to show that $1-t_{x} \leq \frac{1}{2^{p-1}}$. Of course we can suppose that $t_{x}<1$. Let $i \in\left\{0,1, \ldots, 2^{p}-1\right\}$ be such that

$$
\frac{i}{2^{p}} \leq t_{x}<\frac{i+1}{2^{p}}
$$

We necessarily have $i+2>2^{p}$ since otherwise we would get

$$
y \in x X_{p} \subset W_{\frac{i+1}{2 p}} A_{m} W_{\frac{i+1}{2 p}} X_{p} \subset W_{\frac{i+2}{2 p}} A_{m} W_{\frac{i+2}{2 p}} \subset A .
$$

Hence

$$
1-t_{x} \leq 1-\frac{i}{2^{p}}=\frac{2^{p}-i}{2^{p}}<\frac{1}{2^{p-1}} .
$$

Thus we have established that $h \in \mathscr{U}_{L}(G)$. The proof that $h \in \mathscr{U}_{R}(G)$ follows the same way.

Remarks 2.4. (1) Theorem 2.3 obviously implies the following "Urysohn's lemma": let $A$ and $B$ be two subsets of $G$; suppose there exists a neighbourhood $Z$ of $e$ in $G$ such that $Z A Z \cap Z B Z=\varnothing$; then there exists a function $h \in \mathscr{U}(G)$ such that $h(x)=1$ for $x \in A$ and $h(x)=0$ for $x \in B$. Let us remark that the proof of this special case of 2.3 is not easier than the one of 2.3 .

(2) The referee has pointed out that Theorem 2.3 can be generalized to a "Tietze theorem": any bounded bilaterally uniformly continuous function on a subset $A$ of $G$ can be extended to a function $g \in \mathscr{U}(G)$. This can be obtained by making use of the above "Urysohn's lemma" and adapting a standard method which appears in [6, Section 1.17].

\section{IMBEDDING OF $l^{\infty}$ IN $\mathscr{U}_{L}(G) / \mathscr{U}(G)$}

The following lemma, established in the metrizable case, gives the combinatorial core of the proof of the subsequent theorem. 
Lemma 3.1. Let $G$ be a metrizable locally compact topological group and let $V$ be a compact symmetrical neighbourhood of the identity $e$. Let us assume that $\cap_{x \in G} x V x^{-1}$ is not a neighbourhood of $e$ in $G$. Then that

(1) There are two sequences $\left(x_{n}\right)_{n \in \mathscr{N}}$ and $\left(y_{n}\right)_{n \in \mathscr{N}}$ of elements of $G$ such

$$
\lim _{n \rightarrow \infty} x_{n} y_{n}^{-1}=e
$$

for all $m, n \in \mathscr{N}, y_{n}^{-1} x_{m} \notin V$,

for $m \neq n,\left(V y_{m} V^{2}\right) \cap\left(V y_{n} V^{2}\right)=\varnothing$.

(2) Let $g: G \rightarrow[0,2]$ be a continuous function such that $g(e)=2$ and $g(x)=0$ for all $x \in G \backslash V$; let $c=\left(c_{n}\right)_{n \in \mathscr{N}} \in l^{\infty}$. Then we can define the function $f_{c}: G \rightarrow \mathscr{C}$ by

$$
f_{c}(x)=\sum_{n \in \mathscr{N}} c_{n} g\left(y_{n}^{-1} x\right) \quad \text { for all } x \in G,
$$

and $f_{c}$ belongs to $\mathscr{U}_{L}(G)$.

Proof. (1) Let $\left(V_{n}\right)_{n \in \mathscr{N}}$ be a countable decreasing basis of neighbourhoods of $e$ in $G$. For all $n \in \mathscr{N}$, the set $V_{n}$ is not included in $\cap_{y \in G} y V y^{-1}$; hence one can choose $a_{n} \in V_{n}$ and $y_{n} \in G$ such that $a_{n} \notin y_{n} V y_{n}^{-1}$. Let us put $x_{n}=a_{n} y_{n}$, $n \in \mathscr{N}$; then $\lim _{n \rightarrow \infty} x_{n} y_{n}^{-1}=\lim _{n \rightarrow \infty} a_{n}=e$. We have $y_{n}^{-1} x_{n}=y_{n}^{-1} a_{n} y_{n}$ and consequently, for all $n \in \mathscr{N}, y_{n}^{-1} x_{n} \notin V$.

It is immediate that sequences $\left(x_{n}\right)_{n \in \mathscr{N}}$ and $\left(y_{n}\right)_{n \in \mathscr{N}}$ converge to $\infty$. By taking subsequences if necessary, we can suppose that for all $n \in \mathscr{N}$,

$$
x_{n+1}, y_{n+1} \notin\left\{x_{0}, y_{0}, \ldots, x_{n}, y_{n}\right\} V \quad \text { and } y_{n+1} \notin V^{2}\left\{y_{0}, \ldots, y_{n}\right\} V^{4} \text {. }
$$

Hence if $m \neq n$, we have

$$
y_{n}^{-1} x_{m} \notin V \quad \text { and } \quad\left(V y_{m} V^{2}\right) \cap\left(V y_{n} V^{2}\right)=\varnothing .
$$

(2) If $c=0, f_{c}$ is the null function and belongs to $\mathscr{U}_{L}(G)$. Suppose that $c \neq 0$. Since the elements of the sequence $\left(y_{n} V\right)_{n \in \mathcal{N}}$ are pairwise disjoint subsets of $G$, the function $f_{c}$ is well defined; moreover we have $\left|f_{c}(x)\right| \leq 2\|c\|$ for all $x \in G$.

Let us show that $f_{c}$ is left uniformly continuous. Let $\varepsilon>0$ and let us show that there is a neighbourhood $W$ of $e$ in $G$ such that if $x, y \in G$ with $x^{-1} y \in W$, then $\left|f_{c}(x)-f_{c}(y)\right| \leq \varepsilon$.

Since the function $g$ is continuous with a compact support, it is left uniformly continuous. Hence there exists a symmetrical neighbourhood $W \subset V$ of $e$ in $G$ such that for $x, y \in G$,

$$
x^{-1} y \in W \Longrightarrow|g(x)-g(y)| \leq \frac{\varepsilon}{\|c\|} .
$$

Let $x, y \in G$ be such that $x^{-1} y \in W$ and let us show that $\left|f_{c}(x)-f_{c}(y)\right| \leq \varepsilon$. If $x, y \notin \cup_{n \in \mathcal{N}} y_{n} V$, this follows immediately from equalities $f_{c}(x)=f_{c}(y)=0$.

Suppose now that there is $n \in \mathscr{N}$ such that $x \in y_{n} V$; then if $m \neq n$, $x \notin y_{m} V$ because $\left(y_{m} V\right) \cap\left(y_{n} V\right)=\varnothing$; similarly $y \notin y_{m} V$ because $y \in$ $x W \subset y_{n} V W \subset y_{n} V^{2}$ and $\left(y_{m} V\right) \cap\left(y_{n} V^{2}\right)=\varnothing$. Hence it follows from (*) that

$$
\left|f_{c}(x)-f_{c}(y)\right|=\left|c_{n} g\left(y_{n}^{-1} x\right)-c_{n} g\left(y_{n}^{-1} y\right)\right| \leq \varepsilon .
$$


Of course if $y \in y_{n} V$ for some $n \in \mathscr{N}$, we get in the same way that $\mid f_{c}(x)-$ $f_{c}(y) \mid \leq \varepsilon$.

Let us recall that a topological group is called a SIN-group if its two usual uniform structures are equal. It is equivalent to say that for any neighbourhood $V$ of $e$ in $G$ the set $n_{x \in G} x V x^{-1}$ is still a neighbourhood of $e$ in $G$.

We now can state and prove the main result of this paper.

Theorem 3.2. Let $G$ be a locally compact topological group which is not a SINgroup. Then the quotient Banach space $\mathscr{U}_{L}(G) / \mathscr{U}(G)$ contains an isometric linear copy of $l^{\infty}$. In particular $\mathscr{U}_{L}(G) / \mathscr{U}(G)$ is not separable.

Proof. (1) Since $G$ is not a SIN-group, it follows from Corollary 4.5 of [7] (cf. also [13], [14]) that there is a $\sigma$-compact open subgroup $H$ of $G$ which is not a SIN-group. Let $W$ be a neighbourhood of $e$ in $H$ such that $n_{x \in H} x W x^{-1}$ is not a neighbourhood of $e$ in $H$. Let $V$ be a compact symmetrical neighbourhood of $e$ in $H$ such that $V^{2} \subset W$; it follows from the Kakutani-Kodaira's theorem (cf. [9]) that there exists a compact normal subgroup $N$ of $H$ such that $N \subset V$ and such that the quotient $H / N$ is a locally compact metrizable topological group.

For all $x \in H$ let us denote by $\tilde{x}$ the class $x N$ of $H / N$ and for any subset $A \subset H$ let $\tilde{A}=\{\tilde{x} \mid x \in A\}$.

The set $\widetilde{V}$ is a compact symmetrical neighbourhood of $\widetilde{e}$ in $\widetilde{H}$. Let us show that the set $\cap_{x \in H} \widetilde{x} \widetilde{V} \widetilde{x}^{-1}$ is not a neighbourhood of $\widetilde{e}$ in $\widetilde{H}$. Indeed otherwise the set $\cap_{x \in H}\left(x V x^{-1} N\right)$ would be a neighbourhood of $e$ in $H$ but this is false since

$$
\begin{aligned}
\bigcap_{x \in H}\left(x V x^{-1} N\right) & =\bigcap_{x \in H} x V\left(x^{-1} N x\right) x^{-1} \\
& =\bigcap_{x \in H} x(V N) x^{-1} \subset \bigcap_{x \in H} x V^{2} x^{-1} \subset \bigcap_{x \in H} x W x^{-1} .
\end{aligned}
$$

Then it follows from Lemma 3.1 that there exist two sequences $\left(x_{n}\right)_{n \in \mathcal{N}}$ and $\left(y_{n}\right)_{n \in \mathcal{N}}$ of elements of $H$ such that

$$
\begin{gathered}
\lim _{n \rightarrow \infty} \widetilde{x_{n}}{\widetilde{y_{n}}}^{-1}=\widetilde{e}, \\
\text { for all } m, n \in \mathscr{N},{\widetilde{y_{n}}}^{-1} \widetilde{x_{m}} \notin \widetilde{V}, \\
\text { for } m \neq n,\left(\widetilde{V} \widetilde{y_{m}} \widetilde{V}^{2}\right) \cap\left(\widetilde{V} \widetilde{y_{n}} \widetilde{V^{2}}\right)=\varnothing .
\end{gathered}
$$

(2) Let $\widetilde{g}: \widetilde{H} \rightarrow[0,2]$ be a continuous function such that $\widetilde{g}(\widetilde{e})=2$ and $\tilde{g}(\tilde{x})=0$ for all $\tilde{x} \in \tilde{\widetilde{H}} \backslash \widetilde{V}$. Let $c=\left(c_{n}\right)_{n \in \mathcal{N}} \in l^{\infty}$. It follows from Lemma 3.1 that the function $\tilde{f}_{c}: \widetilde{H} \rightarrow \mathscr{C}$ (well) defined by

$$
\tilde{f}_{c}(u)=\sum_{n \in \mathscr{N}} c_{n} \widetilde{g}\left({\widetilde{y_{n}}}^{-1} u\right) \quad \text { for all } u \in \widetilde{H}
$$

belongs to $\mathscr{U}_{L}(\widetilde{H})$.

Let $\phi: G \rightarrow \widetilde{H}$ be the function defined by

$$
\phi(x)= \begin{cases}\tilde{x} & \text { for } x \in H, \\ 0 & \text { for } x \notin H,\end{cases}
$$


and let us put

$$
f_{c}=\tilde{f}_{c} \circ \phi
$$

If $G$ and $\widetilde{H}$ are respectively equipped with their left uniform structures, the function $\phi$ is uniformly continuous. Hence, since $\widetilde{f}_{c}$ belongs to $\mathscr{U}_{L}(\tilde{H})$, the function $f_{c}$ belongs to $\mathscr{U}_{L}(G)$.

(3) Let $\left(N_{k}\right)_{k \in \mathscr{N}}$ be a partition of $\mathscr{N}$ into infinite subsets of $\mathscr{N}$. To any $d=\left(d_{n}\right)_{n \in \mathcal{N}} \in l^{\infty}$ let us associate the sequence $c_{d}=\left(c_{n}\right)_{n \in \mathscr{N}} \in l^{\infty}$ defined by

$$
c_{n}=d_{k} \text { for all } n \in N_{k} \text { and all } k \in \mathscr{N} \text {. }
$$

The function $d \mapsto c_{d}$ is a linear isometry which maps $l^{\infty}$ onto a Banach subspace $E$ of $l^{\infty}$ whose each element $c=\left(c_{n}\right)_{n \in \mathcal{N}}$ satisfies the condition $\limsup _{n \rightarrow \infty}\left|c_{n}\right|=\|c\|$ (let us remark that a similar construction appears in [2]). Consequently to get the theorem, it is sufficient to prove that the linear function

$$
c \mapsto f_{c}+\mathscr{U}(G)
$$

is an isometry from $E$ into the quotient Banach space $\mathscr{U}_{L}(G) / \mathscr{U}(G)$, i.e., that for all $c \in E$

$$
\inf \left\{\left\|f_{c}+h\right\| \mid h \in \mathscr{U}(G)\right\}=\|c\| .
$$

Let $c=\left(c_{n}\right)_{n \in \mathcal{N}} \in E$ and let $h \in \mathscr{U}(G)$; let us show that $\left\|f_{c}+h\right\| \geq\|c\|$. Let $\left(\left|c_{n_{p}}\right|\right)_{p \in \mathscr{N}}$ be a subsequence of $\left(\left|c_{n}\right|_{n \in \mathcal{N}}\right)$ such that

$$
\lim _{p \rightarrow \infty}\left|c_{n_{p}}\right|=\limsup _{n \rightarrow \infty}\left|c_{n}\right|=\|c\| \text {. }
$$

It follows from (1) that $\lim _{p \rightarrow \infty} \widetilde{x_{n_{p}}}{\widetilde{y_{n_{p}}}}^{-1}=\tilde{e}$; hence, if $p$ is big enough, $\widetilde{x_{n_{p}}}{\widetilde{y_{n_{p}}}}^{-1} \in \tilde{V}$ or equivalently $x_{n_{p}} y_{n_{p}}^{-1}$ belongs to the compact subset $V N$ of $H$. Let $\left(x_{n_{\alpha}} y_{n_{\alpha}}^{-1}\right)$ be a subnet of $\left(x_{n_{p}} y_{n_{p}}^{-1}\right)$ which converges in $H$ to a point $z$; we have

$$
\widetilde{z}=\lim _{\alpha} \widetilde{x_{n_{\alpha}}} \widetilde{y_{n_{\alpha}}}-1=\tilde{e}
$$

For any $\alpha$ we have

$$
\begin{aligned}
& \left|\left(f_{c}+h\right)\left(x_{n_{\alpha}}\right)-\left(f_{c}+h\right)\left(z^{-1} y_{n_{\alpha}}\right)\right| \\
& \quad \geq\left|f_{c}\left(x_{n_{\alpha}}\right)-f_{c}\left(z^{-1} y_{n_{\alpha}}\right)\right|-\left|h\left(x_{n_{\alpha}}\right)-h\left(z^{-1} y_{n_{\alpha}}\right)\right| .
\end{aligned}
$$

It follows from the definition of $\left(x_{n}\right),\left(y_{n}\right)$, and $f_{c}$ (cf. (2), (3), and (4)) and from (5) that

$$
\left|f_{c}\left(x_{n_{\alpha}}\right)-f_{c}\left(z^{-1} y_{n_{\alpha}}\right)\right|=\left|\widetilde{f_{c}}\left(\widetilde{y_{n_{\alpha}}}\right)\right|=2\left|c_{n_{\alpha}}\right| ;
$$

hence

$$
\lim _{\alpha}\left|f_{c}\left(x_{n_{\alpha}}\right)-f_{c}\left(z^{-1} y_{n_{\alpha}}\right)\right|=2\|c\| .
$$

By the definition of $z$

$$
\lim _{\alpha} x_{n_{\alpha}}\left(z^{-1} y_{n_{\alpha}}\right)^{-1}=e,
$$

and consequently, since $h \in \mathscr{U}(G)$, we have

$$
\lim _{\alpha}\left|h\left(x_{n_{\alpha}}\right)-h\left(z^{-1} y_{n_{\alpha}}\right)\right|=0
$$


It follows from (6), (7), and (8) that

$$
\limsup _{\alpha}\left|\left(f_{c}+h\right)\left(x_{n_{\alpha}}\right)-\left(f_{c}+h\right)\left(z^{-1} y_{n_{\alpha}}\right)\right| \geq 2\|c\|
$$

which implies that $\left\|f_{c}+h\right\| \geq\|c\|$.

To conclude it is now sufficient to prove that for all $c \in E$ there exists $h_{1} \in \mathscr{U}(G)$ such that $\left\|f_{c}+h_{1}\right\| \leq\|c\|$. Since $\left(\widetilde{V} \widetilde{y_{n}} \widetilde{V}^{2}\right)_{n \in \mathcal{N}}$ is a sequence of pairwise disjoint subsets of $\widetilde{H}$, it follows from Theorem 2.3 that there exists a function $\widetilde{h_{1}} \in \mathscr{U}(\widetilde{H})$ such that for all $n \in \mathscr{N}$

$$
\widetilde{h_{1}}(u)=-c_{n} \quad \text { if } u \in \widetilde{y_{n}} \widetilde{V},
$$

and such that $\left\|\widetilde{h_{1}}\right\|=\|c\|$. Let $u \in \widetilde{H}$; if $u \notin \cup_{n \in \mathcal{N}} \widetilde{y_{n}} \widetilde{V}$, we have

$$
\left|\left(\widetilde{f_{c}}+\widetilde{h_{1}}\right)(u)\right|=\left|\widetilde{h_{1}}(u)\right| \leq\left\|\widetilde{h_{1}}\right\|=\|c\| ;
$$

if $u \in \widetilde{y_{n}} \widetilde{V}$ for some $n \in \mathscr{N}$, then

$$
\left|\left(\widetilde{f}_{c}+\widetilde{h_{1}}\right)(u)\right|=\left|c_{n}\right|\left|\widetilde{g}\left({\widetilde{y_{n}}}^{-1} u\right)-1\right| \leq\left|c_{n}\right| \leq\|c\|
$$

and therefore $\left\|\tilde{f}_{c}+\widetilde{h_{1}}\right\| \leq\|c\|$.

Let $h_{1}: G \rightarrow \mathscr{C}$ be the function $h_{1}=\widetilde{h_{1}} \circ \phi$; since $\widetilde{h_{1}} \in \mathscr{U}(\widetilde{H})$ and since $\phi$ is uniformly continuous when $G$ and $\widetilde{H}$ are equipped with their left (respectively right) uniformity, the function $h_{1}$ belongs to $\mathscr{U}(G)$; moreover

$$
\left\|f_{c}+h_{1}\right\|=\left\|\tilde{f}_{c} \circ \phi+\widetilde{h_{1}} \circ \phi\right\|=\left\|\left(\tilde{f}_{c}+\widetilde{h_{1}}\right) \circ \phi\right\|=\left\|\tilde{f}_{c}+\widetilde{h_{1}}\right\|,
$$

and consequently $\left\|f_{c}+h_{1}\right\| \leq\|c\|$.

Remark 3.3. The referee has pointed out that another imbedding theorem is also true: $l^{\infty} \backslash\{0\}$ can be isometrically imbedded into $\mathscr{U}_{L}(G) \backslash \mathscr{U}(G)$. The proof of this statement is easy by making use of the results of [10].

\section{REFERENCES}

1. J. F. Berglund, H. D. Junghenn, and P. Milnes, Analysis on semigroups: Function spaces, compactifications, representations, Wiley, New York, 1989.

2. C. Chou, Weakly almost periodic functions and almost convergent functions on a group, Trans. Amer. Math. Soc. 206 (1975), 175-200.

3. W Weakly almost periodic functions and Fourier-Stieljes algebras of locally compact groups, Trans. Amer. Math. Soc. 274 (1982), 141-157.

4. H. A. M. Dzinotyiweyi, Nonseparability of quotient spaces of function algebras on topological semigroups, Trans. Amer. Math. Soc. 272 (1982), 223-235.

5. __ Uniformly continuous and weakly almost periodic functions on some topological semigroups, Proc. Amer. Math. Soc. 114 (1992), 571-574.

6. L. Gillman and M. Jerison, Rings of Continuous Functions, Univ. Ser. Higher Math., Van Nostrand Reinhold, Princeton, NJ, 1960.

7. G. Hansel and J. P. Troallic, Equicontinuity, uniform structures and countability in locally compact groups, Semigroup Forum 42 (1991), 167-173.

8. E. Hewitt and K. A. Ross, Abstract harmonic analysis. I, Springer-Verlag, New York, 1963.

9. G. L. Itzkowitz, Continuous measures, Baire category, and uniform continuity in topological groups, Pacific J. Math. 54 (1974), 115-125.

10. G. L. Itzkowitz, S. Rothman, H. Strassberg, and T. S. Wu, Characterization of equivalent uniformities in topological groups, Topology Appl. 47 (1992), 9-34. 
11. M. Katetov, On real-valued functions in topological spaces, Fund. Math. 38 (1951), 85-91.

12. _ Correction to "On real-valued functions in topological spaces", Fund. Math. 40 (1953), 203-205.

13. P. Milnes, Uniformity and uniformly continuous functions for locally compact groups, Proc. Amer. Math. Soc. 109 (1990), 567-570.

14. V. G. Pestov, A test of balance of a locally compact group, Ukrainian Math. J. 40 (1988), 109-111.

URA CNRS D1378, Faculté des Sciences, Université de Rouen, F-76134 Mont-SaintAIGNAN, France

E-mail address: Georges.Hansel Clitp.ibp.fr

URA CNRS D1378, Faculté des Sciences et des Techniques, Université du Havre, 25, rue Philippe Lebon, F-76600 le HaVre, France

E-mail address: troallicefst.univ-lehavre.fr 